\title{
The Anti-Cancer Effect of Pitavastatin May Be a Drug-Specific Effect: Subgroup Analysis of the TOHO-LIP Study
}

\author{
Daiji Nagayama iD ${ }^{1,2}$ \\ Atsuhito Saiki ${ }^{2}$ \\ Kohji Shirai ${ }^{3}$ \\ 'Department of Internal Medicine, \\ Nagayama Clinic, Tochigi, Japan; ${ }^{2}$ Center \\ of Diabetes, Endocrine and Metabolism, \\ Toho University Sakura Medical Center, \\ Chiba, Japan; ${ }^{3}$ Department of Internal \\ Medicine, Mihama Hospital, Chiba, Japan
}

Correspondence: Daiji Nagayama Department of Internal Medicine, Nagayama Clinic, 2-12-22, Tenjin-Cho, Oyama-City, Tochigi, 323-0032, Japan $\mathrm{Tel} / \mathrm{Fax}+8 \mathrm{I}-285-22-0219$

Email deverlast9607।@gmail.com

\begin{abstract}
The significance of statin treatment for the reduction of cardiovascular (CV) disease has been reported, whereas other reports have also described anti-cancer properties associated with the class effect of statins. However, the differences in anti-cancer effect of various types of statins have rarely been examined. Pitavastatin is a statin with a different chemical structure and pharmacokinetics from other statins, and the mechanism of the specific anti-cancer effect of pitavastatin has been reported in in vivo therapeutic models. We previously revealed that pitavastatin therapy was superior to atorvastatin therapy in the prevention of CV events, despite similar LDL-cholesterol-lowering effect in the TOHO Lipid Intervention Trial Using Pitavastatin (TOHO-LIP). Furthermore, in subgroup analysis of the TOHO-LIP study, cumulative 240 -week incidence of new cancer cases tended to be lower in the pitavastatin group compared to the atorvastatin group [0.32\% (1/312) vs $1.94 \%(6 / 310)$, log-rank $\mathrm{P}=0.051]$. This finding might reveal the superiority of pitavastatin to prevent carcinogenesis. The molecular mechanism by which pitavastatin suppresses the incidence of any-organ cancer is gradually elucidated, and new combination of cancer treatments with pitavastatin will be developed in the future to further enhance the anti-cancer activity and reduce the side effects.
\end{abstract} Keywords: pitavastatin, anti-cancer effect, TOHO-LIP

\section{Statins as Anti-Cancer Agents}

Hydroxymethylglutaryl (HMG)-CoA reductase inhibitors, also known as statins, inhibit the production of mevalonic acid from HMG-CoA, leading to upregulation of hepatic low-density lipoprotein (LDL) receptor and resulting in potent LDLcholesterol-lowering effect. ${ }^{1}$ Moreover, the significance of statin treatment for the reduction of cardiovascular $(\mathrm{CV})$ disease has been reported. ${ }^{2,3}$ On the other hand, the BioBank Japan cohort study including 41,930 Japanese patients has indicated that statin treatment could potentially reduce mortality related to any-organ and colorectal cancer. ${ }^{4}$ Other reports have described anti-cancer properties associated with the class effect of statins, through inhibiting the HMG-CoA reductase pathway as well as cellular proliferation, migration, and survival of cancer cells by regulating Rho, Ras, and Rac proteins. ${ }^{5}$ Statins have been demonstrated to inhibit Ras prenylation and downstream signaling of Ras, including extracellular signalregulated kinase $1 / 2$ (ERK1/2), Akt, and mammalian target of rapamycin (mTOR), thereby inducing apoptosis of cancer cells through suppression of geranylgeranyl diphosphate (GGPP) biosynthesis. ${ }^{6}$ However, the differences in anticancer effect of various types of statins have rarely been examined. 


\section{Combined Pitavastatin and} Capmatinib Enhances Tumor Growth Inhibition in in vivo Therapeutic Model

Pitavastatin is a statin with a different chemical structure and pharmacokinetics from other statins, and has been clinically available in Japan since 2003 for the treatment of primary hyperlipidemia or mixed dyslipidemia. ${ }^{7}$ The long-term safety and efficacy of pitavastatin is considered to be due to its low metabolism by CYP enzymes, resulting in minimal drug-drug interactions. ${ }^{8}$ Recently, $\mathrm{Xu}$ et $\mathrm{al}^{9}$ have reported the mechanism of the specific anti-cancer effect of pitavastatin in in vivo therapeutic models using oral and esophageal squamous cell carcinoma (OSCC and ESCC) cells. According to this report, pitavastatin inhibits tumor growth by downregulating AKT and ERK signals. In in vivo therapeutic models, pitavastatin enhanced the tumor growth inhibitory effect of capmatinib, a mesenchymal-epithelial transition (MET) specific inhibitor. In general, cancer cells invade basement membranes and metastasize to distant sites through MET. This study may provide new insight of the epigenetic change induced by pitavastatin as a sensitizer to overcome resistance to capmatinib monotherapy. Thus, research on the use of HMG-CoA reductase inhibitors, including pitavastatin, in cancer treatment is constantly evolving, and new combination therapies are expected in the future. Meanwhile, despite the promising basic research results, there are not enough reports on the status of carcinogenesis in daily clinical practice.

\section{TOHO-LIP Study Compared Pitavastatin and Atorvastatin}

Atorvastatin is one of the most commonly used statins worldwide, and has been established to be effective for primary and secondary prevention of $\mathrm{CV}$ events in hypercholesterolemic patients. ${ }^{10}$ We previously conducted a multicenter, open-label, randomized controlled trial of head-to-head comparison between pitavastatin and atorvastatin, named the TOHO Lipid Intervention Trial Using Pitavastatin (TOHO-LIP). ${ }^{11}$

This trial, which analyzed 622 hypercholesterolemic patients (mean age 65 years) with one or more CV risks, revealed that pitavastatin ( $2 \mathrm{mg} /$ day $)$ treatment was superior to atorvastatin $(10 \mathrm{mg} /$ day $)$ treatment in the prevention of the primary end point which was a composite of CV death, sudden death of unknown origin, nonfatal myocardial infarction, nonfatal stroke, transient ischemic attack and heart failure requiring hospitalization, despite similar LDL-cholesterol-lowering effect of both statins. Furthermore, a subgroup analysis of TOHO-LIP suggests that increased preheparin (nonheparinized) serum level of lipoprotein lipase (LPL) mass by pitavastatin treatment may be associated with the preventive effect on $\mathrm{CV}$ events. ${ }^{12}$ We next conducted another subgroup analysis of the TOHO-LIP to compare the effects of both statins on carcinogenesis. This analysis showed that cumulative 240 -week incidence of new cancer cases tended to be lower in the pitavastatin group compared to the atorvastatin group $[0.32 \%(1 / 312)$ vs $1.94 \%(6 / 310)$, log-rank $\mathrm{P}=0.051]$, as shown in Figure 1. The new cancer cases were as follows: (pitavastatin group) hepatocellular carcinoma 1, (atorvastatin group) lung carcinoma 2, hepatocellular carcinoma 1, prostate carcinoma 1, renal cell carcinoma 1 and gastric carcinoma 1 . This finding revealed that pitavastatin treatment might be superior to atorvastatin treatment also for the prevention of carcinogenesis.

\section{Molecular Biological Mechanisms for the Anticancer Effects of Pitavastatin}

LPL, which is anchored on the surface of vascular endothelium and hydrolyzes triglyceride in the blood, is mainly produced in adipocytes and skeletal muscle cells. ${ }^{13}$ We previously reported that pitavastatin enhances the expression of LPL protein and mRNA through activating adenosine monophosphate-activated protein kinase (AMPK) in skeletal muscle cells. AMPK activation is a pharmacological effect that is also shared by aspirin and metformin, ${ }^{14}$ which have been shown to have anticancer effects. ${ }^{15,16}$ Furthermore, the role of AMPK in suppressing MET by modulating the Akt signaling has been reported. ${ }^{17}$ When considered in conjunction with the aforementioned report showing interference with MET signaling by pitavastatin, ${ }^{7}$ the relationship between AMPK and MET may partially explain the effect of pitavastatin in inhibiting carcinogenesis. Additionally, one of the major downstream signaling pathways regulated by AMPK is the mTOR pathway, which is consistently deregulated in cancer cells. ${ }^{18}$

Previous studies have revealed that pitavastatin induces apoptosis through the activation of caspases in in vitro and inhibits tumor growth in in vivo xenograft models in 


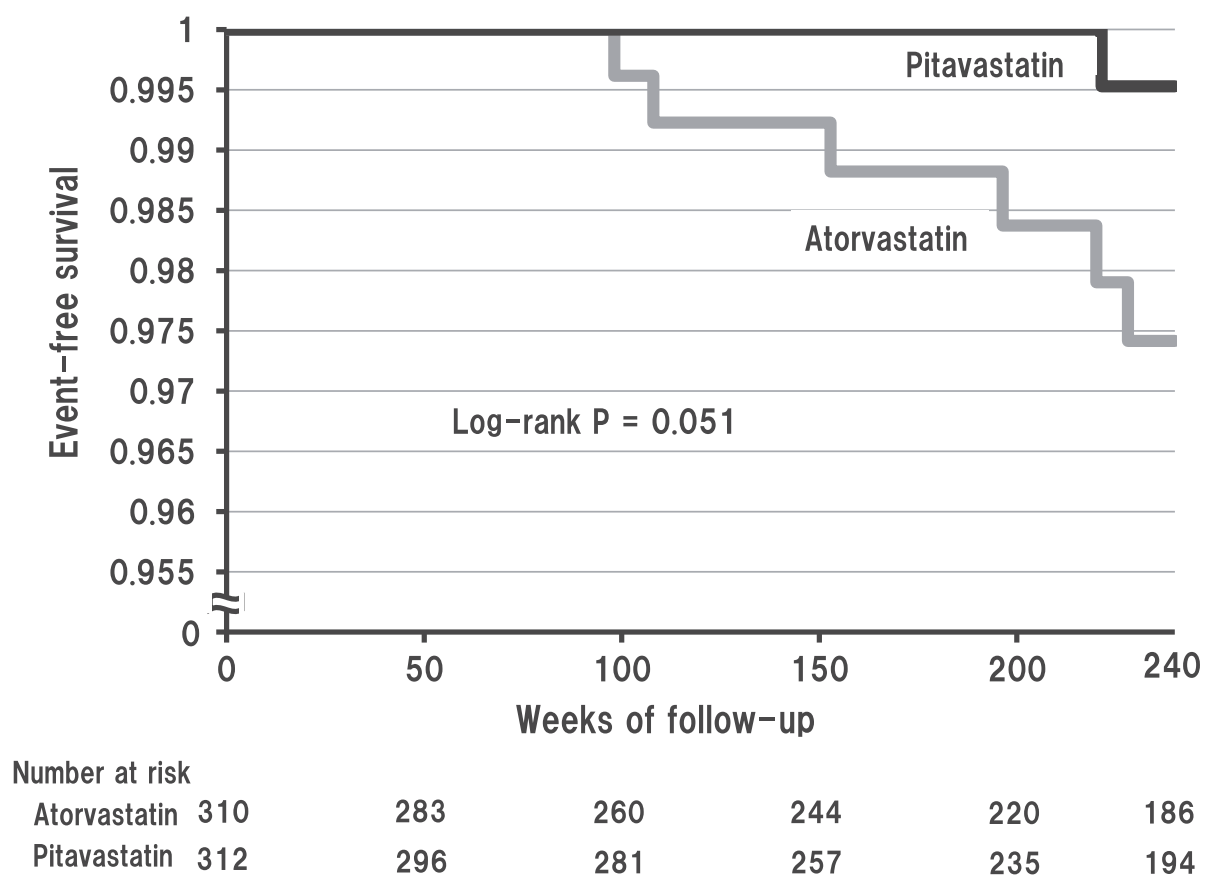

Figure I Incidence of new cancer cases in two statin groups. Kaplan-Meier curves for new cancer cases.

OSCC and ESCC, glioblastoma, breast, liver, colon, ovarian and pancreatic cancers. ${ }^{19-25}$ These reports demonstrated that the effects of pitavastatin on the cell cycle arrest were enhanced through the upregulation of cell cycle regulators such as p53, p27 and p21. Furthermore, the activation of caspase- $3 / 7$ by pitavastatin and the promotion of expression of proapoptotic Bcl-2 family members support the notion that pitavastatin mediates apoptosis through the intrinsic apoptotic pathway. On a molecular level, pitavastatin treatment has also been demonstrated to upregulate the cell cycle regulator p21 and to inhibit nuclear factor $-\kappa \mathrm{B}(\mathrm{NF}-\kappa \mathrm{B})$ in different cancer cells, which resulted in cell cycle arrest and apoptosis. ${ }^{26}$

The mechanisms of epigenetics have been shown to be deeply involved in the etiology of cancer. On the other hand, inhibition of the mevalonate pathway by statins directly or indirectly affects cancer cell growth, differentiation and apoptosis through epigenetic mechanisms. As described earlier, it has been revealed that the suppression of the MET signaling pathway via Golgi apparatus dysfunction caused by pitavastatin was important for tumor growth inhibition in OSCC and ESCC, ${ }^{7}$ although other specific epigenetic change induced by pitavastatin as a sensitizer to overcome resistance to chemotherapy was not yet fully understood. On the other hand, nonepigenetic pathways modulated by pitavastatin have been reported. Otahal et al demonstrated the promising cytotoxic effects of the combination of pitavastatin and erlotinib using an EGFR-TKI-resistant human lung adenocarcinoma cell line in which EGFR is mutated or overexpressed. ${ }^{27}$ In addition, Jiao et al found that administration of pitavastatin to human MCF10A, one of the non-malignant breast epithelial cells, resulted in depletion of GGPP in the mevalonate pathway and starvation of amino acids, eventually leading to cell death. ${ }^{28}$

\section{Conclusion}

Pitavastatin treatment reduced the incidence of new cancer cases more efficaciously than atorvastatin treatment despite similar LDL-cholesterol-lowering effect of the two statins in the TOHO-LIP study. The molecular mechanism by which pitavastatin suppresses the incidence of any-organ cancer is gradually elucidated, and new combination cancer treatments with pitavastatin will be developed in the future to further enhance the anti-cancer activity and reduce the side effects.

\section{Ethical Statement}

The protocol of the TOHO-LIP study was prepared in accordance with Declaration of Helsinki and approved by the Ethics Committee of Toho University Sakura Medical Center (S18069). Informed consent was obtained in the form of opt-out on the website. 


\section{Acknowledgment}

The authors appreciate the researchers and participants of TOHO-LIP study, and are grateful to Dr. Hideaki Nitta (Hematology/Oncology Division, Tokyo Medical University, Tokyo, Japan) for valuable advice.

\section{Disclosure}

The authors report no conflicts of interest in this manuscript.

\section{References}

1. Corsini A, Bellosta S, Baetta R, et al. New insights into the pharmacodynamic and pharmacokinetic properties of statins. Pharmacol Ther. 1999;84(3):413-428. doi:10.1016/S0163-7258(99)00045-5

2. Okazaki S, Yokoyama T, Miyauchi K, et al. Early statin treatment in patients with acute coronary syndrome: demonstration of the beneficial effect on atherosclerotic lesions by serial volumetric intravascular ultrasound analysis during half a year after coronary event: the ESTABLISH study. Circulation. 2004;110(9):1061-1068. doi:10.1161/01.CIR.0000140261.58966.A4

3. Nakamura H, Arakawa K, Itakura H, et al.; MEGA Study Group. Primary prevention of cardiovascular disease with pravastatin in Japan (MEGA Study): a prospective randomized controlled trial. Lancet. 368;2006:1155-1163. doi:10.1016/S0140-6736(06)69472-5

4. Yokomichi H, Nagai A, Hirata M, et al. Statin use and all-cause and cancer mortality: bioBank Japan cohort. $J$ Epidemiol. 2017;27(3S): S84-S91. doi:10.1016/j.je.2016.12.011

5. Di Bello E, Zwergel C, Mai A, Valente S. The innovative potential of statins in cancer: new targets for new therapies. Front Chem. 2020;8:516. doi: $10.3389 /$ fchem. 2020.00516

6. Tsubaki M, Fujiwara D, Takeda T. The sensitivity of head and neck carcinoma cells to statins is related to the expression of their Ras expression status, and statin-induced apoptosis is mediated via suppression of the Ras/ERK and Ras/mTOR pathways. Clin Exp Pharmacol Physiol. 2017;44(2):222-234. doi:10.1111/14401681.12690

7. Saito Y. Pitavastatin: an overview. Atheroscler Suppl. 2011;12 (3):271-276. doi:10.1016/S1567-5688(11)70886-8

8. Saito Y. Critical appraisal of the role of pitavastatin in treating dyslipidemias and achieving lipid goals. Vasc Health Risk Manag. 2009;5:921-936. doi:10.2147/VHRM.S5551

9. Xu B, Muramatsu T, Inazawa J. Suppression of MET signaling mediated by pitavastatin and capmatinib inhibits oral and esophageal cancer cell growth. Mol Cancer Res. 2020;19(4):585-597.

10. Sever PS, Dalhoff B, Poulter NR, et al. Prevention of coronary and stroke events with atorvastatin in hypertensive patients who have average or lower-than-average cholesterol concentrations, in the Anglo-Scandinavian Cardiac Outcomes Trial-Lipid Lowering Arm (ASCOT-LLA): a multicenter randomised controlled trial. Lancet. 2003;361(9364):1149-1158. doi:10.1016/S0140-6736(03)12948-0

11. Moroi M, Nagayama D, Hara F, et al. Outcome of pitavastatin versus atorvastatin therapy in patients with hypercholesterolemia at high risk for atherosclerotic cardiovascular disease. Int $J$ Cardiol. 2020;305:139-146. doi:10.1016/j.ijcard.2020.01.006

12. Nagayama D, Saiki A, Watanabe Y, et al. Prevention of cardiovascular events with pitavastatin is associated with increased serum lipoprotein lipase mass level: subgroup analysis of the TOHO-LIP. J Atheroscler Thromb. 2021. doi:10.5551/jat.62141
13. Nilsson-Ehle PA, Garfinkel AS, Schotz MC. Lipolytic enzymes and plasma lipoprotein metabolism. Annu Rev Biochem. 1991;49:667-693. doi:10.1146/annurev.bi.49.070180.003315

14. Ohira M, Endo K, Saiki A, et al. Atorvastatin and pitavastatin enhance lipoprotein lipase production in L6 skeletal muscle cells through activation of adenosine monophosphate-activated protein kinase. Metabolism. 2012;61(10):1452-1460. doi:10.1016/j. metabol.2012.03.010

15. Din FVN, Valanciute A, Houde VP, et al. Aspirin inhibits mTOR signaling, activates AMP-activated protein kinase, and induces autophagy in colorectal cancer cells. Gastroenterology. 2012;142(7):15041515.e3. doi:10.1053/j.gastro.2012.02.050

16. Yue W, Yang CS, DiPaola RS, Tan X-L. Repurposing of metformin and aspirin by targeting AMPK-mTOR and inflammation for pancreatic cancer prevention and treatment. Cancer Prev Res. 2014;7 (4):388-397. doi:10.1158/1940-6207.CAPR-13-0337

17. Chou CC, Lee KH, Lai IL, et al. AMPK reverses the mesenchymal phenotype of cancer cells by targeting the Akt-MDM2-Foxo3a signaling axis. Cancer Res. 2014;74(17):4783-4795. doi:10.1158/00085472.CAN-14-0135

18. Okubo K, Isono M, Miyai K, Asano T, Sato A. Fluvastatin potentiates anticancer activity of vorinostat in renal cancer cells. Cancer Sci. 2020;111(1):112-126. doi:10.1111/cas. 14225

19. Lee N, Pun N, Jang W, Bae J, Jeong C. Pitavastatin induces apoptosis in oral squamous cell carcinoma through activation of FOXO3a. J Cell Mol Med. 2020;24(12):7055-7066. doi:10.1111/jcmm.15389

20. Jiang P, Mukthavaram R, Chao Y, et al. In vitro and in vivo anticancer effects of mevalonate pathway modulation on human cancer cells. $\mathrm{Br}$ J Cancer. 2014;111(8):1562-1571. doi:10.1038/bjc.2014.431

21. You HY, Zhang WJ, Xie XM, Zheng ZH, Zhu HL, Jiang FZ. Pitavastatin suppressed liver cancer cells in vitro and in vivo. Onco Targets Ther. 2016;9:5383-5388. doi:10.2147/OTT.S106906

22. Zhang ZY, Zheng SH, Yang WG, Yang C, Yuan WT. Targeting colon cancer stem cells with novel blood cholesterol drug pitavastatin. Eur Rev Med Pharmacol Sci. 2017;21:1226-1233.

23. de Wolf E, Abdullah MI, Jones SM, et al. Dietary geranylgeraniol can limit the activity of pitavastatin as a potential treatment for drug-resistant ovarian cancer. Sci Rep. 2017;7(1):5410. doi:10.1038/ s41598-017-05595-4

24. Villarino N, Signaevskaia L, van Niekerk J, et al. A screen for inducers of bHLH activity identifies pitavastatin as a regulator of $\mathrm{p} 21, \mathrm{Rb}$ phosphorylation and E2F target gene expression in pancreatic cancer. Oncotarget. 2017;8(32):53154-53167. doi:10.18632/ oncotarget. 18587

25. Wang L, Wang Y, Chen A, et al. Pitavastatin slows tumor progression and alters urine-derived volatile organic compounds through the mevalonate pathway. FASEB J. 2019;33(12):13710-13721. doi:10.1096/fj.201901388R

26. Fujino M, Miura S, Matsuo Y, Tanigawa H, Kawamura A, Saku K. Pitavastatin-induced downregulation of CCR2 and CCR5 in monocytes is associated with the arrest of cell-cycle in $\mathrm{S}$ phase. Atherosclerosis. 2006;187(2):301-308. doi:10.1016/j. atherosclerosis.2005.10.008

27. Otahal A, Aydemir D, Tomasich E, Minichsdorfer C. Delineation of cell death mechanisms induced by synergistic effects of statins and erlotinib in non-small cell lung cancer cell (NSCLC) lines. Sci Rep. 2020;10(1):959. doi:10.1038/s41598-020-57707-2

28. Jiao Z, Cai H, Long Y, et al. Statin-induced GGPP depletion blocks macropinocytosis and starves cells with oncogenic defects. Proc Natl Acad Sci U S A. 2020;117(8):4158-4168. doi:10.1073/ pnas. 1917938117 


\section{Publish your work in this journal}

Vascular Health and Risk Management is an international, peerreviewed journal of therapeutics and risk management, focusing on concise rapid reporting of clinical studies on the processes involved in the maintenance of vascular health; the monitoring, prevention and treatment of vascular disease and its sequelae; and the involvement of metabolic disorders, particularly diabetes. This journal is indexed on PubMed Central and MedLine. The manuscript management system is completely online and includes a very quick and fair peerreview system, which is all easy to use. Visit http://www.dovepress. com/testimonials.php to read real quotes from published authors.

Submit your manuscript here: https://www.dovepress.com/vascular-health-and-risk-management-journal 